Reprod. Nutr. Dévelop., 1988, 28 Suppl. n¹, 159-160

\title{
Mécanismes impliqués dans la réaction allergique intestinale aux protéines de soja chez le veau préruminant
}

Christine DUVAUX, J. W. SISSONS $\left({ }^{1}\right)$, Lesley HEPPELL $\left({ }^{1}\right)$, R. TOULLEC, P. GUILLOTEAU

Laboratoire du Jeune Ruminant,

I.N.R.A., 65, rue de Saint-Brieuc, 35042 Rennes Cedex, France.

( $\left.{ }^{1}\right)$ Pig Nutrition and Production Department,

A.F.R.C., I.G.A.P., Church Lane, Shinfield, Reading RG2 9AO, U.K.

Summary. Studies of intestinal motility in calves given antigenic soya protein or sucrose showed disturbances linked with diarrhoea. Disorders arising from feeding antigenic soya protein were distinct from abnormal motility induced by undigestible carbohydrate. Suppression of the digestive disorders by a drug having anti-allergic properties implied the involvement of an immunological mechanism.

Les veaux préruminants nourris avec un lait de remplacement contenant du tourteau de soja présentent des troubles digestifs et une croissance faible (Sissons et Smith, 1976). L'augmentation parallèle du titre d'anticorps antiprotéines de soja, suggère le développement d'une réaction allergique gastrointestinale. Cependant, le tourteau de soja contient également des oligosides indigestibles dans l'intestin grêle qui sont susceptibles d'entraîner des diarrhées osmotiques. Le but de ce travail a été d'étudier l'implication des anticorps (IgG et/ou $\lg E$ ) et des oligosides dans les troubles observés.

Matériel et méthodes. Quatre veaux âgés d'un mois, munis d'une canule abomasale et de 5 groupes d'électrodes sur la surface séreuse de l'intestin grêle, depuis le duodénum jusqu'à l'iléon proximal, ont reçu par infusion intrapéritonéale un sérum provenant de veaux nourris au soja. Ce sérum était riche en IgG anti-soja mais ses $\operatorname{lgE}$ avaient été inactivées par chauffage à $56^{\circ} \mathrm{C}$ pendant $30 \mathrm{~min}$. Des enregistrements de la motricité intestinale ont été réalisés après des repas d'épreuve de $2,7 \mathrm{~kg}$ de laits de remplacement dont les protéines étaient apportées par de la caséine (aliment $\mathrm{C}$ ) ou du tourteau de soja cuit (aliment $\mathrm{S}$ ). Ces aliments étaient infusés dans la caillette en 1 à $2 \mathrm{~min}$, tous les 2 ou $3 \mathrm{j}$. Après l'apparition de désordres dans la motricité intestinale accompagnés de diarrhée, les animaux ont été traités durant $3 \mathrm{j}$ avec 2 ou $4 \mathrm{~g} / \mathrm{j}$ d'un médicament anti-allergique [Nedocromil sodium (Fisons plc)] avant de recevoir à nouveau l'aliment S. Enfin, $200 \mathrm{~g}$ de saccharose ont été ajoutés à l'aliment C. Des échantillons de sang ont été prélevés régulièrement dans une veine jugulaire pour mesurer les titres d'IgG anti-soja par hémagglutination passive.

Résultats et discussion. Malgré l'apparition d'un titre élevé d'IgG anti-soja à la suite de l'infusion du sérum, le premier repas d'aliment $S$ n'entraîne pas de modifications dans la motricité intestinale par comparaison avec l'aliment $C$ (fig. $1 \mathrm{a}, \mathrm{b}$ ). En revanche, après plusieurs repas d'aliment $\mathrm{S}$ (en moyenne à partir du cinquième), des désordres moteurs caractérisés par un nombre de plus en plus 
élevé de complexes myoélectriques migrants (MMC) (fig. 1c) se propageant très rapidement et s'accompagnant d'une diarrhée, apparaissent. Ces désordres moteurs sont donc liés à l'apparition de diarrhée mais ne semblent pas être provoqués par la présence des IgG anti-soja. L'addition de saccharose à l'aliment $\mathrm{C}$ conduit aussi à l'apparition d'une diarrhée, mais le profil myoélectrique où prédomine l'activité irrégulière (fig. 1d), bien que différent de celui obtenu avec l'aliment $C$ sans saccharose, n'est en aucune façon semblable au profil anormal observé avec l'aliment $\mathrm{S}$. Les désordres moteurs provoqués par l'aliment $S$ ne semblent donc pas provenir d'un effet osmotique dû aux oligosides indigestibles présents dans le soja. Après le traitement des veaux par le médicament anti-allergique, l'aliment $S$ n'entraîne plus de désordres moteurs ni de diarrhée (fig. 1e). Ceci suggère que les troubles observés auparavant avec l'aliment $S$ étaient dus à une réaction d'hypersensibilité immédiate, d'autant plus que les désordres moteurs apparaissaient rapidement (moins de $30 \mathrm{~min}$ ) après le repas. De plus, il a été montré que des veaux réagissant fortement produisent des IgE anti-soja (Kilshaw et Sissons, 1979) et que le nombre de mastocytes augmente dans leur paroi intestinale (Pedersen, 1986).

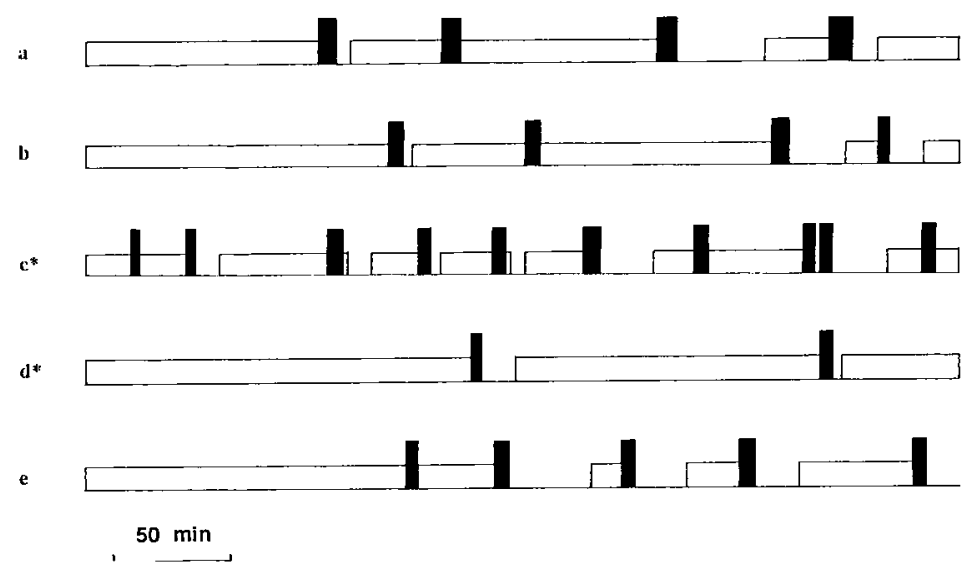

FIG.1. - Exemples de profils moteurs enregistrés au niveau du jéjunum moyen au cours des 6 premières heures suivant l'infusion abomasale de l'aliment $C$ (a), de l'aliment $S$ juste après l'infusion de sérum (b), de l'aliment $S$ après l'apparition des désordres (c), de l'aliment $C$ additionné de saccharose (d) et de l'aliment $S$ après traitement anti-allergique (e).

*: présence de diarrhée ; $\square$. I: phases d'activités irrégulière et régulière du MMC.

En conclusion, l'intolérance aux protéines de soja chez le veau est due à une réaction allergique intestinale impliquant une réaction immunitaire immédiate conduisant à l'apparition de désordres moteurs caractéristiques sur l'intestin grêle.

Sissons J. W., Smith R. H., 1976. Br. J. Nutr., 36, 421-438.

Sissons J. W. et al., 1987. In Chandra R. K., Food allergy, 95-108, Nutr. Res. Educ. Fdn, St Johns, Newfoundland.

Pedersen H. E., 1986. PhD Thesis. Reading Univ., U.K., 323 p.

Kilshaw P. J., Sissons J. W., 1979. Res. Vet. Sci., 27, 361-365. 